\title{
POBLAMIENTO E INTENSIDAD DE USO DEL ESPACIO EN LA CUENCA DEL LAGO SAN MARTIN (PATAGONIA ARGENTINA): NUEVOS DATOS DESDE LA MARGEN NORTE
}

\section{PEOPLEMENT AND LANDSCAPE INTENSITY USE AT SAN MARTÍN LACUSTRINE BASIN (PATAGONIA ARGENTINA): NEW DATA FROM THE NORTH MARGIN}

\author{
Silvana Espinosa ${ }^{1}$, Juan Bautista Belardi ${ }^{2}$, Gustavo Barrientos ${ }^{3}$ y \\ Carballo Marina, Flavia ${ }^{4}$ \\ ${ }^{1}$ CONICET - UNPA, Campus Universitario, Piloto Rivera y Av. Gregores, (9400) Río Gallegos, Santa Cruz. \\ E-mail: silvanaespinosa@conicet.gov.ar \\ ${ }^{2}$ UNPA - CONICET Campus Universitario, Piloto Rivera y Av. Gregores, (9400) Río Gallegos, Santa Cruz. E-mail: \\ juanbautistabelardi@gmail.com \\ ${ }^{3}$ CONICET - FCNyM - UNLP. E-mail: gustavbarrie@yahoo.com.ar \\ ${ }^{4} \mathrm{UNPA}$, Campus Universitrario, Piloto Rivero y Av. Gregores, (9400) Río Gallegos, Santa Cruz. \\ E-mail: flaviacarballomarina@gmail.com
}

Presentado el: 31/12/2012 - Aceptado 19/04/2013

\section{Resumen}

La arqueología de las poblaciones cazadoras-recolectoras de las cuencas lacustres Tar y San Martín (Santa Cruz, Argentina) señala diferencias en la forma e intensidad de uso del espacio. En este trabajo se incorporan nuevos datos tecnológicos y cronológicos de la distribución de artefactos de la margen norte del lago San Martín, obtenidos en transectas y excavaciones en abrigos rocosos ubicados en la estepa y en el ecotono bosque-estepa. Los resultados alcanzados permiten completar el paisaje arqueológico de los alrededores de este lago e incorporarlo a la discusión regional, reforzando planteos previos acerca de la baja intensidad de uso humano del espacio y la articulación logística de los sectores occidentales, cercanos al borde de bosque, desde espacios ubicados hacia el este.

Palabras claves: Cuencas lacustres pericordilleranas, tecnología lítica, cronología, intensidad de uso del espacio.

Abstract

The archaeological record of the hunter-gatherer populations that inhabited the Tar and San Martin lacustrine basins during the Holocene indicates differences in both the mode and the intensity in which these two spaces were used. In this paper we contribute with new data about spatial artifact distribution, lithic technology and the occupation chronology of the northern margin of the San Martin lake that were obtained in transect surveys and excavations carried out in rockshelters situated in 
steppe and steppe-wood ecotonal environments. The obtained results allow both the construction of a model about the circum-lake archaeological landscape and the incorporation of this space into the regional archaeological discussion, further supporting previous observations about the low intensity of the human use of this space and the logistic utilization of the western sectors of the basin, near the current edge of the forest, from occupation centers situated eastward.

Keywords: Peri-Andean lacustrine basins, lithic technology, radiocarbon chronology, space-use intensity

\section{Introducción}

Los lagos Tar y San Martín ocupan una cuenca glaciaria situada en el suroeste de la Patagonia Argentina (Provincia de Santa Cruz) ( $\left.49^{\circ} 7^{\prime} \mathrm{S}, 72^{\circ} 11^{\prime} \mathrm{O}\right)$, en un sector pericordillerano a 240 m.s.n.m. El lago San Martín, ubicado hacia el oeste, continúa hacia el lado chileno con el nombre de lago O’Higgins.

Las investigaciones arqueológicas sistemáticas iniciadas en el año 2006, centralizadas inicialmente en el lago Tar y en la margen sur del lago San Martín, produjeron un primer cuerpo de datos que indicaron diferencias en la forma e intensidad en el uso del espacio por parte de las poblaciones cazadoras-recolectoras durante el Holoceno (Belardi et al. 2009, 2010; Belardi y Campan 2009; Espinosa et al. 2009; Pallo 2009).

A continuación, se resumirán brevemente las características ambientales de la región, así como las inferencias realizadas a partir de los primeros trabajos llevados a cabo en la cuenca de estos lagos. Posteriormente, se presentará nueva información distribucional, tecnológica y cronológica obtenida, la que será discutida desde una perspectiva regional.

\section{Ambiente y paleoambiente}

Las cuencas de los lagos Tar y San Martín tienen una orientación noroeste-sudeste. Están circundadas por diferentes sistemas de morrenas, producto del modelado glaciario (Auer y Cappannini 1957; Bonarelli y Nágera 1921; Rabassa y Coronato 2002). Se ubican próximas al Campo de Hielo Sur, que las limita por el oeste mientras que, por el norte, lo hace la meseta del Cardiel-Chico y, por el sur, las mesetas que las comunican con el lago Viedma (Figura 1). A diferencia del lago Tar, el San Martín no conforma un espacio abierto sino que se encuentra encajonado entre mesetas altas y la cordillera.

Cabe mencionar que estas cuencas se ubican en el extremo oeste del eje de circulación natural que es el valle del río Chalía, que corre en sentido NO-SE. Asimismo, en dirección N-S, están próximas al corredor donde se encuentra la actual Ruta Nacional $\mathrm{N}^{\circ} 40$, otro importante eje de circulación. Por último, en la margen este del lago Tar, desembocan los ríos Tar y Meseta (al norte y sur, respectivamente) que también constituyen rutas naturales que unen la cuenca con la meseta del Cardiel Chico y del lago Viedma (Belardi et al. 2009).

En la región bajo análisis, las unidades ecológicas presentes son la estepa y el ecotono bosque-estepa. En la actualidad, la estepa graminosa abarca la mayor porción de la margen norte del lago San Martín, incluyendo sectores de la península Chacabuco y la bahía La Lancha. Asimismo, parte de estas dos últimas lo ocupa un estrecho ecotono bosque-estepa y bosque (caracterizado por la presencia de Nothofagus). La creciente desertificación de la 
región (Auer y Cappannini 1957) determina buenas condiciones de visibilidad arqueológica en la estepa, estimada sobre el porcentaje de cobertura vegetal por $\mathrm{m}^{2}$. Resultan así cuatro categorías: muy buena (0-25\%), buena (26-50 \%), regular (51-75 \%) y mala (76-100 \%). Las condiciones de visibilidad de la estepa favorecieron la implementación de una metodología distribucional para la recuperación del registro arqueológico.

Se han realizado estudios sobre registros polínicos fósiles de una turbera localizada en la estancia La Tercera, margen sur del lago San Martín. Los análisis de polen y de partículas de carbón indican condiciones de extrema aridez, con muy bajas precipitaciones entre los 11.300 y 10.000 años cal AP. Entre los 10.000 y 8000 años cal. AP. en dicha cuenca dominó una estepa graminosa, sugiriendo un incremento en la disponibilidad de humedad por un aumento en las precipitaciones. Esto se relaciona con la expansión del bosque de Nothofagus en la zona andina, evidenciando condiciones más cálidas y húmedas (Bamonte 2012).

Antes de los 7540 años radiocarbónicos AP., se habría dado una vegetación asociada con precipitaciones entre 500 y $300 \mathrm{~mm}$.; posteriormente y hasta los 5700 años AP., decreció la humedad con precipitaciones que oscilaron entre 400 y $200 \mathrm{~mm}$. Luego, durante unos 200 años, aumentó la humedad (representando un ambiente que carece de análogo actual). Por último, en el período comprendido entre 5500 y 4000 años AP., existió una menor disponibilidad hídrica (Bamonte y Mancini 2009). Alrededor de los 3000 años cal. AP., nuevamente disminuye la humedad (Bamonte 2012).

Este estudio fue complementado con el análisis de una secuencia polínica obtenida en el mallín del Paisano Desconocido (Bamonte 2012), ubicado sobre el sector noreste de Bahía La Lancha, margen norte del lago San Martín. La secuencia se inicia hacia 6650 años cal. AP. Entre esa fecha y los 5150 años cal. AP, el ambiente se corresponde con una estepa arbustiva, sugiriendo condiciones más secas que las actuales. Hacia el 6000 años cal. AP los valores altos de Nothofagus indican el inicio del bosque en la zona andina y en la Península Chacabuco. Entre los 5000 y los 4000 años cal. AP, se observa una mayor densidad de arbustos y una disminución abrupta de los pastos. Por otra parte, hacia los 4000 años cal. AP, la señal del bosque desciende de manera marcada. Los cambios más significativos en la secuencia suceden entre esta fecha y los 3000 años cal. AP, produciéndose el desarrollo de la estepa graminosa como consecuencia de un aumento de la humedad mientras que, en la zona andina, disminuyen los valores de Nothofagus, situación que se mantiene hasta los 100 años cal. AP. A partir de este momento, se observan condiciones más secas (Bamonte 2012).

Para los últimos 2000 años cal. AP, el estudio de sedimentos del fondo del lago del Desierto - ubicado inmediatamente al oeste del lago San Martín - indica que, hacia 850 años cal. AD, se inició la Anomalía Climática Medieval (ACM), un período más cálido que el actual que culmina en torno a los 1450 años cal. AD (Kastner et al. 2010). Luego, el ambiente se torna más frío y húmedo durante la Pequeña Edad del Hielo, que dura hasta 1750 años $\mathrm{AD}$ (Masiokas et al. 2009).

\section{La información arqueológica previa}

De acuerdo con las investigaciones en la margen sur del lago San Martín, se propone un uso intermitente y pautado de la región, desde ca. 11.100 años cal. AP - fechado correspondiente al sitio Bloque 1-Oquedad -, bajo condiciones muy secas, inmediatamente después 
de la retirada de los glaciares (Bamonte 2012). Luego de un marcado hiato entre $c a .7600$ y 5600 años cal. AP, se reconocen nuevas ocupaciones.

Las edades radiocarbónicas correspondientes a los niveles con ocupación de los sitios de la margen sudeste del lago San Martín, al igual que las obtenidas en la margen este del lago Tar y en el cerro Cach Aike, señalan una mayor intensidad de las ocupaciones para el Holoceno tardío (últimos 2500 años cal. AP.) reflejando una integración plena de los distintos ambientes de estas cuencas.

Así, en la estepa, los sectores de fondo de cuenca provistos de lugares protegidos como los médanos habrían sido ocupados durante todo el año. Tal el caso de la margen este del lago Tar y de los alrededores del Cerro Cach Aike, dos espacios que han sido centrales en el uso humano a través del tiempo y en relación con la circulación de bienes y poblaciones (Belardi et al. 2009, 2010). Se suma a ello que la obtención de las materias primas líticas habría sido relativamente sencilla, dada la alta disponibilidad y fácil acceso a las mismas, tanto en afloramientos rocosos como en lechos de los ríos y en las mesetas circundantes. Se han detectado filones de toba silicificada verde en el istmo de la península Maipú, en la margen sur del lago San Martín (Espinosa et al. 2009). Por el contrario, en su margen norte, no se reconocieron fuentes de aprovisionamiento de materia prima, aunque en términos potenciales podría contemplarse el arroyo de las Minas y los ríos Fósiles y Caracoles. Por otra parte se han reconocido, tanto en contextos al aire libre como en abrigos rocosos y desde momentos iniciales del poblamiento de la cuenca, dos rocas alóctonas. Una de ellas es la obsidiana, que macroscópicamente es semejante a la registrada en la Pampa del Asador (Espinosa y Goñi 1999; Stern 1999), distante 150 Km. al NE del lago San Martín. La otra es la limolita, disponible sobre la margen oeste del lago Cardiel (Belardi et al. 2003). Ambas son indicadores directos de la conexión con los espacios ubicados hacia el norte.

La evidencia sugiere que la intensidad en el uso de estos distintos espacios decrece desde el este hacia el oeste, sustentando la hipótesis de marginalidad regional (Borrero 2004). Esto cobra sentido al considerar que, los espacios más cercanos a la cordillera, tienen una estacionalidad más marcada. Así, desde el sector más occidental hasta ahora investigado - el istmo de la península Maipú, caracterizado por un ambiente de ecotono - se habrían articulado partidas logísticas. Por otra parte, se conforma aquí un "fondo de saco", ya que la transitabilidad hacia el oeste se ve dificultada por la vegetación boscosa, la topografía abrupta y la cercanía del Campo de Hielo Sur.

La margen sur del lago San Martín presenta evidencias de un uso logístico, menos intensivo y permanente en contraposición con aquellos sectores localizados al este, principalmente en los alrededores del lago Tar. La circulación de rocas empleadas para la talla se ajusta a esta descripción ya que, por ejemplo, la obsidiana está presente en todos los espacios mencionados pero disminuye sus frecuencias hacia el oeste. Por el contrario, la toba silicificada verde cuando se presenta en contextos hacia el este, lo hace en muy baja frecuencia.

La presencia de obsidiana "limolita" y de valvas marinas, junto con la información provista por motivos rupestres permiten proponer rutas de circulación de bienes y poblaciones (Belardi et al. 2009), que articulan la región de estudio con las cuencas de los lagos Cardiel y Viedma (ubicadas hacia el norte y sur respectivamente). 


\section{Metodología}

El abordaje arqueológico de la cuenca se realizó desde una perspectiva espacial, a través del análisis distribucional de materiales arqueológicos por medio de la implementación de transectas (Dunnell y Dancey 1983; ver Belardi et al. 2010 para la región de estudio). Las transectas permitieron obtener frecuencias artefactuales, que se usaron para el cálculo de densidades por $\mathrm{m}^{2}$. Por otra parte, se evaluó el porcentaje de muestreos sin hallazgos con el propósito de reconocer las diferencias en la intensidad de depositación de artefactos en un espacio determinado.

La intensidad de las ocupaciones se analizó sobre la base de las densidades artefactuales y a partir de las tasas de depositación de materiales arqueológicos. Estas últimas se obtienen dividiendo el total de desechos de talla con talón, núcleos y artefactos formatizados, por la datación disponible local o regionalmente. La misma se puede descomponer en diferentes períodos (por ejemplo y, tal como aquí se hace, cada 100 años). Este acercamiento, eminentemente comparativo, permite establecer si habría habido un descarte diferencial con respecto a la frecuencia de artefactos (ver Borrero 1993; Belardi 2005). En este trabajo y de manera exploratoria para posibilitar la comparación entre los reparos rocosos que aquí se presentan, se suponen semejantes las condiciones sedimentarias durante el lapso considerado (ver Barberena 2008, discusión allí planteada).

El análisis espacial se complementa con la información radiocarbónica y con el estudio tecnológico, llevado a cabo tanto sobre muestras obtenidas al aire libre como aquellas provenientes de excavaciones. Se siguieron los lineamientos tecnomorfológicos propuestos por C. Aschero (1975 rev. 1983) y se tomaron en consideración el estado de conservación, la clase artefactual, las materias primas y los tamaños de los desechos de talla enteros.

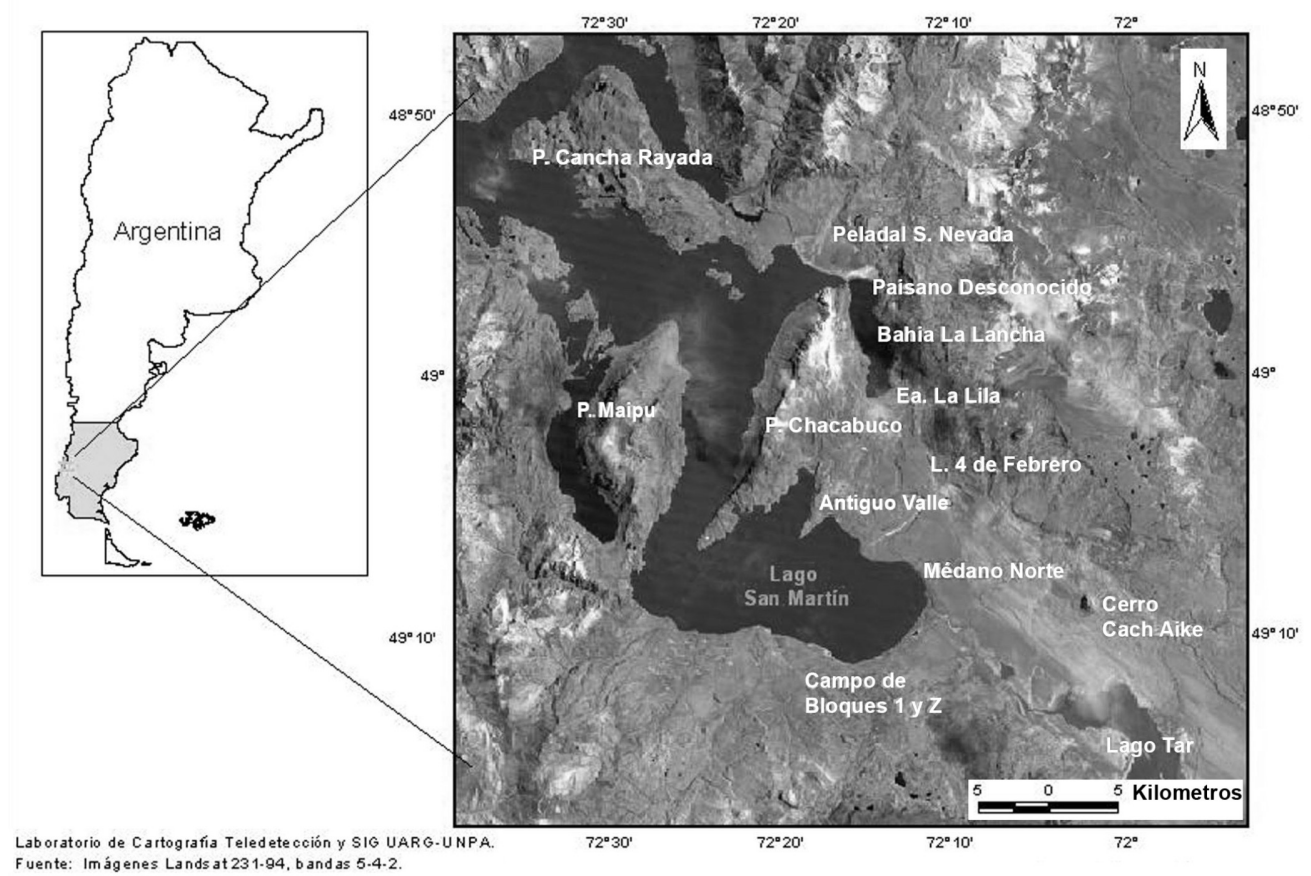

Figura 1.- Margen norte del blago San Martín. Sectores analizados 
La margen norte del lago San Martín

Esta margen del lago San Martín (Figura 1) comprende un importante sector de estepa, aún en la bahía La Lancha y en la península Chacabuco. En esta última, también se ha relevado el ecotono y el borde de bosque. En la Tabla 1 se presentan los sitios según la unidad ecológica en la que se encuentran, ordenados de este a oeste.

\begin{tabular}{|c|c|}
\hline Estepa & Ecotono (bosque - estepa) \\
\hline Laguna 4 de Febrero & Península Chacabuco (Borde de bosque) \\
\hline \multirow[t]{14}{*}{ Medano Margen norte } & Lagunas 1 y 2 \\
\hline & Sitio Mallín del Puesto de Turba \\
\hline & Bosquetes (transectas) \\
\hline & Bahía La Lancha. Cabeceras \\
\hline & Btoque Errático Ea. La Lita \\
\hline & Pampay Antiguo Valle (d̈esagüe) \\
\hline & Terraza Intermedia \\
\hline & Bahía La Lancha Margen noroeste \\
\hline & Lagunas \\
\hline & Alero del Paisano Desconociäo \\
\hline & Transecta del Camino \\
\hline & Cueva del Paisano Desconociäo \\
\hline & Peladal Sierra Nevada \\
\hline & Istmo Península Cancha Rayada \\
\hline
\end{tabular}

labla 1. Sectores analızados por unidad ecológica.

En la estepa, se relevó la laguna denominada 4 de Febrero (340 m.s.n.m.). Se recolectaron materiales en cinco concentraciones, que totalizaron $858 \mathrm{~m}^{2}$; de donde provienen artefactos líticos, dos guijarros de obsidiana negra (Belardi et al. 2009) y un tiesto cerámico decorado.

Entre la Laguna 4 de Febrero y el lago San Martín, se extiende una amplia planicie glaciaria (345 m.s.n.m.) sobre la que se realizaron transectas (Tabla 2). Las mismas alcanzaron la costa acantilada del lago, donde se ubicó el sitio Médano Margen norte. Los materiales líticos y restos óseos de guanaco se encontraban en cuatro hoyadas de deflación contiguas, que cubrían una superficie de $345 \mathrm{~m}^{2}$. Cabe destacar el hallazgo de una chaquira sobre valva. Más hacia el este, la planicie glaciaria está disectada por el antiguo valle de desagüe de la bahía La Lancha. El desnivel es de alrededor de $70 \mathrm{~m}$. Sobre una terraza intermedia se registró el sitio Bahía La Lancha; los materiales cubrían una superficie de $600 \mathrm{~m}^{2}$.

En la península Chacabuco, se realizaron transectas sobre el borde de bosque y en bosquetes aislados, sin producirse hallazgos (Tabla 2). Sobre el sector noreste de la península se relevaron las denominadas Lagunas 1 y 2 . En la margen sur de la primera laguna, se recolectaron materiales sobre una superficie de $1500 \mathrm{~m}^{2}$. Por otra parte, en el extremo Este de un mallín situado en una cicatriz de erosión de $128 \mathrm{~m}^{2}$, se recuperaron artefactos en una concentración que se denominó Mallín del Puesto de Turba. Todos los materiales reseñados se encuentran al este de la línea de bosque; la Laguna 1 es la más próxima a manchones de bosque aislados (bosquetes), ubicados a unos $200 \mathrm{~m}$ hacia el oeste de la misma. 
Se exploró la cabecera de bahía La Lancha y no se registraron hallazgos en superficie. Se realizó una cuadrícula de $1 \mathrm{~m}^{2}$ en un bloque denominado Bloque Errático de Ea. La Lila, donde se recuperó una muy baja frecuencia de materiales (Tabla 2). Hacia el oeste, sólo se prospectó la margen noreste de la bahía, ya que la margen opuesta - ubicada sobre la península Chacabuco - cae en pendiente hacia el agua. Sobre la ruta provincial $N^{\circ} 31$, que es la ruta de circulación natural ya que los cerros del norte enmarcan la bahía, en bajos estructurales de la Fm. Bahía La Lancha se recorrieron siete lagunas someras. En cuatro de ellas se produjeron hallazgos, distribuidos en una superficie de $1619 \mathrm{~m}^{2}$ (Tabla 2).

En el extremo noreste de la bahía, en el ecotono bosque-estepa, y ubicados sobre un extenso mallín, se registraron tres abrigos rocosos que presentaban materiales arqueológicos en superficie. En dos de ellos, llamados Alero del Paisano Desconocido y Cueva del Paisano Desconocido respectivamente, se llevaron a cabo recolecciones de artefactos y excavaciones de $1 \mathrm{~m}^{2}$. Debe destacarse que en la ladera opuesta (península Chacabuco), la vegetación corresponde a un bosque de Nothofagus.

El Alero del Paisano Desconocido (427 m.s.n.m) se encuentra bajo un bloque desprendido de otro mayor, perteneciente a la Fm. Chon Aike. Se halla orientado al sur y posee un ancho máximo de 5,80 $\mathrm{m}$ y una profundidad de 2,70 $\mathrm{m}$. En la estratigrafía no se observan bloques colapsados ni desprendimientos de magnitud, sino sólo guijarros en el nivel más profundo de la excavación (-1 m). La Cueva del Paisano Desconocido se encuentra a $700 \mathrm{~m}$ del Alero, a mayor altura, poseyendo un ancho de boca de $14 \mathrm{~m}$ y una profundidad de 16,9 $\mathrm{m}$, alcanzando, en su parte más protegida, una altura de 2,10 m. En ambos sitios predominan las arqueofaunas de guanaco (Lama guanicoe), las que se encuentran bajo análisis. Los nuevos datos cronológicos se obtuvieron sobre huesos de guanaco - provenientes de ambos sitios - con indicios de procesamiento antrópico (Tabla 3). Esta información se complementa con la procedente de una transecta efectuada directamente sobre la ruta provincial $\mathrm{N}^{\circ} 31$, con el fin de privilegiar la visibilidad arqueológica (Tabla 2).

\begin{tabular}{|c|c|c|c|c|c|}
\hline N Transectas & $\begin{array}{c}\mathrm{N} \\
\text { muestreos }\end{array}$ & $\begin{array}{c}\text { Superficie } \\
\mathrm{m}^{2}\end{array}$ & $\begin{array}{c}\text { Ny } \% \\
\text { muestreos } \\
\text { con } \\
\text { hallazgos }\end{array}$ & $\begin{array}{c}\mathrm{N} \text { artefactos } \\
\text { / densidades } \\
\mathrm{m}^{2}\end{array}$ & Visibilidad \\
\hline \multicolumn{6}{|c|}{$\begin{array}{l}\text { Planicie entre laguna } 4 \text { de Febrero y margen norte del lago San Martín y del antiguo } \\
\text { valle de desagüe de bahía La Lancha }\end{array}$} \\
\hline 8 & 130 & 174500 & $10 / 7,69$ & $13 / 0,00007$ & Muy Buena \\
\hline \multicolumn{6}{|c|}{ Línea de bosque y bosquetes. Península Chacabuco } \\
\hline 2 & 17 & 17000 & 0 & 0 & $\begin{array}{l}\text { Regular- } \\
\text { Buena }\end{array}$ \\
\hline \multicolumn{6}{|c|}{ Cabecera de bahía La Lancha } \\
\hline 1 & 10 & 25000 & 0 & 0 & Buena \\
\hline \multicolumn{6}{|c|}{ Paisano Desconocido (frente al alero y la cueva). Sobre ruta provincial $\mathrm{N}^{\circ} 31$} \\
\hline 1 & 1 & 1843 & $1 / 100$ & $25 / 0,0004$ & Muy buena \\
\hline \multicolumn{6}{|c|}{ Peladal Sierra Nevada } \\
\hline 1 & 20 & 2000 & $1 / 5$ & $1 / 0,0005$ & Muy buena \\
\hline \multicolumn{6}{|c|}{ Istmo de la Península Cancha Rayada } \\
\hline 1 & 25 & 12500 & $2 / 8$ & $2 / 0,0001$ & Muy buena \\
\hline
\end{tabular}

Tabla 2. Información de transectas. 


\begin{tabular}{|l|l|l|l|}
\hline Alero del Paisano Desconocido \\
\hline $\begin{array}{l}\text { Nivel } \\
\text { estratigráfico }\end{array}$ & $\begin{array}{l}\text { Elemento datado } \\
\text { (Lama guanicoe) }\end{array}$ & $\begin{array}{l}\text { Datación } \\
\text { (años AP.) }\end{array}$ & Laboratorio \\
\hline$-20-30 \mathrm{~cm}$ & 1 falange proximal & $380 \pm 25$ & UGAMS 3550 \\
\hline$-55-60 \mathrm{~cm}$ & $\begin{array}{l}\text { Metatarso proximal + } \\
\text { diáfisis }\end{array}$ & $680 \pm 25$ & UGAMS 3551 \\
\hline$-101 \mathrm{~cm}$ & Húmero distal + diáfisis & $4720 \pm 30$ & UGAMS 3552 \\
\hline Cueva del Paisano Desconocido & $\begin{array}{l}\text { Datación } \\
\text { Nivel } \\
\text { estratigráfico }\end{array}$ & Lamanto datado & Laboratorio \\
\hline$-35 \mathrm{~cm}$ & Radiocúbito proximal & $3030 \pm 30$ & UGAMS 4671 \\
\hline$-40 \mathrm{~cm}$ & Costilla proximal & $4040 \pm 30$ & UGAMS 4672 \\
\hline$-68,5-71 \mathrm{~cm}$ & Escápula & $6930 \pm 40$ & UGAMS 2945 \\
\hline$-80-85 \mathrm{~cm}$ & Metatarso proximal & $8000 \pm 40$ & UGAMS 4673 \\
\hline
\end{tabular}

Tabla 3. Dataciones radiocarbónicas obtenidas por AMS (sin calibrar) de los sitios excavados en bahía La Lancha. Todos los elementos datados poseen huellas de actividad antrópica (corte, fracturas longitudinales y percusión).

\begin{tabular}{|l|l|l|l|l|l|l|l|}
\hline \multirow{2}{*}{ Proveniencia } & \multirow{2}{*}{$\begin{array}{c}\text { Artefactos } \\
\text { fommatiza dos* }\end{array}$} & \multicolumn{5}{|c|}{ Desechos de talla } & \multirow{2}{*}{ Total } \\
\cline { 3 - 8 } & & Chunk & LENT & LFCT & LFST & INDI & \\
\hline $\begin{array}{l}\text { Laguna 4 de } \\
\text { Febrero }\end{array}$ & 47 & 2 & 89 & 45 & 76 & 6 & 265 \\
\hline $\begin{array}{l}\text { Médano Margen } \\
\text { norte }\end{array}$ & 9 & 0 & 18 & 12 & 13 & 2 & 54 \\
\hline Pla. Chacabuco & 12 & 0 & 5 & 2 & 16 & 0 & 35 \\
\hline Bahía La Lancha & 15 & 0 & 17 & 17 & 10 & 0 & 59 \\
\hline $\begin{array}{l}\text { Bloque Errático } \\
\text { Ea. La Lila }\end{array}$ & 0 & 0 & 1 & 2 & 3 & 1 & 7 \\
\hline $\begin{array}{l}\text { Alero Paisano } \\
\text { Desconocido }\end{array}$ & 21 & 5 & 393 & 254 & 394 & 28 & 1095 \\
\hline $\begin{array}{l}\text { Cueva Paisano } \\
\text { Desconocido }\end{array}$ & 8 & 0 & 17 & 11 & 21 & 3 & 60 \\
\hline $\begin{array}{l}\text { Peladal Sierra } \\
\text { Nevada }\end{array}$ & 0 & 0 & 1 & 0 & 0 & 0 & 1 \\
\hline $\begin{array}{l}\text { Istmo Pla. } \\
\text { Cancha Rayada }\end{array}$ & 2 & 0 & 0 & 0 & 0 & 0 & 2 \\
\hline TOTAL & 114 & 7 & 541 & 343 & 533 & 40 & 1578 \\
\hline
\end{tabular}

Tabla 4. Frecuencias de artefactos formatizados y desechos de talla según los espacios relevados. Ref.: LENT: lasca entera; LFCT: lasca fracturada con talón; LFST: lasca fracturada sin talón; INDI: indiferenciada. * Sólo se consignan artefactos manufacturados, no se incluyen guijarros que han sido sumados al total de las piezas contabilizadas. 
Por último, el registro más occidental de artefactos en la margen norte del lago San Martín corresponde a un extenso espacio denudado llamado Peladal Sierra Nevada y al extremo noreste del istmo de la Península Cancha Rayada (Tabla 1).

\section{Tecnología lítica}

La muestra alcanza un total de 1549 piezas que se distribuyen a lo largo de los diferentes espacios relevados (Tabla 4).

El estado de conservación de los desechos de talla es bueno, garantizando la confiabilidad de la muestra; 885 conservan el talón, lo que alcanza el 61,33\%, mientras que 559 no lo poseen, constituyendo el $38,66 \%$ de los casos.

En el grupo de los artefactos formatizados, la relación se invierte, superando las piezas fracturadas $(\mathrm{N}=74 ; 64,9 \%)$ a las enteras $(\mathrm{N}=40 ; 35 \%)$. Esta situación es diferente a lo registrado en otros sectores de la cuenca de los lagos Tar y San Martín y en otros sitios estratificados de la región. En el caso del Alero del Paisano Desconocido, puede deberse a una alteración en la depositación sedimentaria (Súnico, comunicación personal a J. B. B. 2010).

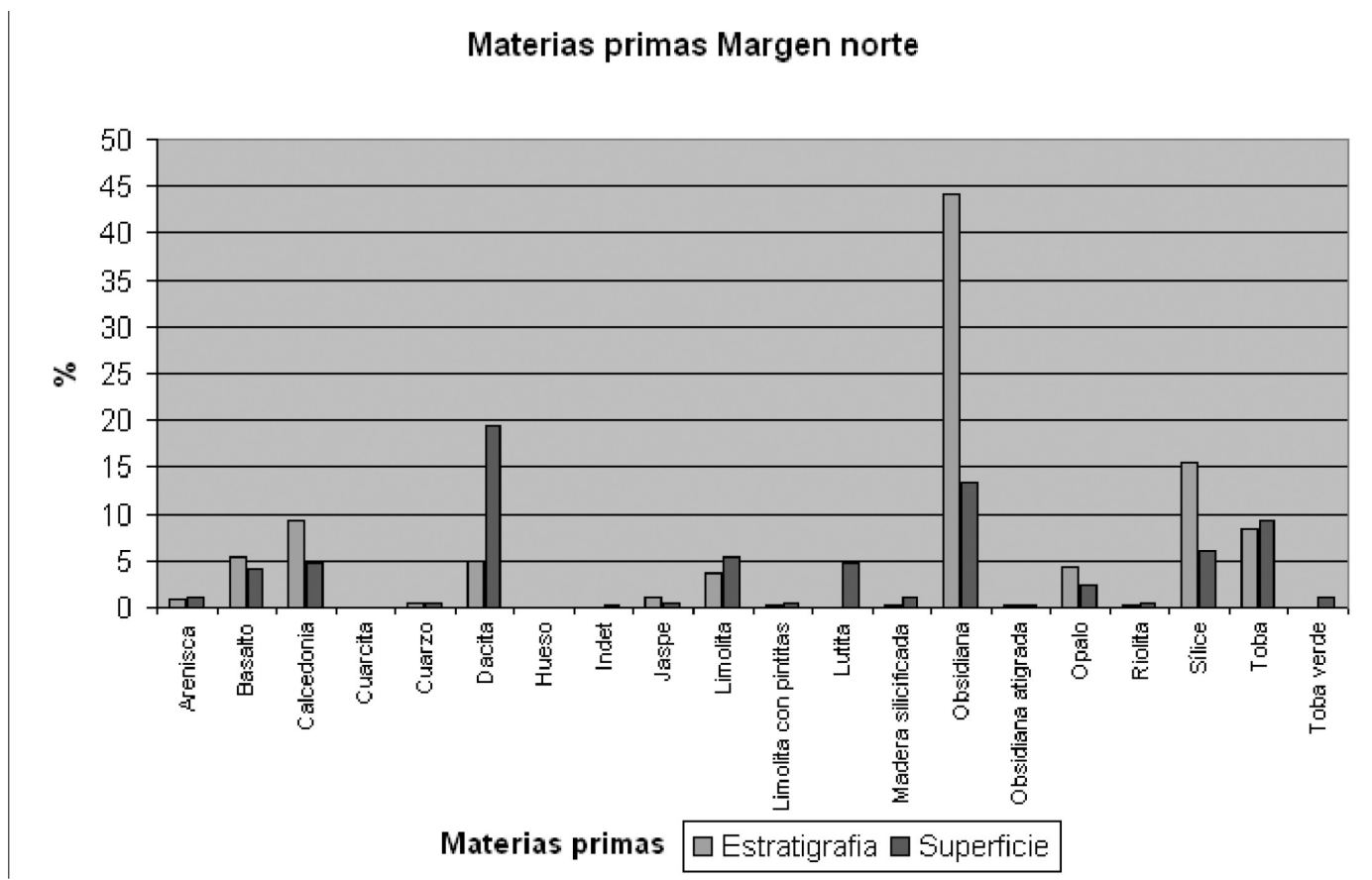

Figura 2.- Distribución de materias primas de los artefactos formatizados y desechos de talla.

En la Figura 2 se observa la amplia variedad de rocas utilizadas en la manufactura de artefactos, destacándose la obsidiana, la dacita, la sílice, la limolita y la toba.

Se destaca la alta frecuencia de piezas de obsidiana, principalmente desechos de talla, además de piezas bifaciales y raspadores. Esta roca sólo resulta superada, en los sitios de superficie, por la dacita. Asimismo, la diversidad de clases artefactuales en esta última es superior a la obsidiana; entre ellas, núcleos, cuchillos y raederas. Sin embargo, es muy bajo el índice de bifacialidad $(0,05)$, mientras que entre los artefactos de obsidiana es alto $(0,1)$. Esta 
clara tendencia refleja un uso sumamente específico de esta última roca; se trata, entonces, de una tecnología conservada, orientada a la confiabilidad de las piezas en una actividad fundamental como puede ser la caza.

La composición de los conjuntos de dacita hace suponer su disponibilidad local, principalmente por la presencia de núcleos y desechos con claras evidencias de trabajos iniciales de manufactura. Asimismo, entre los artefactos manufacturados en toba, también se destacan los núcleos $(\mathrm{N}=8)$ y un $3 \%$ de desechos con restos de corteza. Las características expeditivas de los artefactos formatizados subraya esta tendencia.

Con respecto a la sílice, se diferencia de las otras rocas presentadas ya que las clases artefactuales se reducen a un núcleo, un biface, un filo natural con rastros complementarios (FNCR) y nueve raspadores. A su vez, es muy baja la presencia de corteza $(1,8 \%)$, siendo los tipos de desechos mayoritarios los internos y de reactivación, de tamaños muy pequeños, por lo cual no se espera la realización de las primeras etapas de la manufactura. La trayectoria de esta roca en los sitios analizados habría incluido el uso y consecuente reactivación de los artefactos, cuya formatización habría ocurrido en otro sector.

En líneas generales, la muestra de los desechos de talla presenta altas frecuencias, alcanzando un total de 1443 (93,64\%). En sitios bajo abrigo, de acuerdo con sus tamaños, se observa que la mayoría se ubica en los intervalos menores (Tablas 5 y 6). Esto indicaría la realización casi exclusiva de las últimas etapas de manufactura y reactivación de artefactos. Esto se evidencia más claramente en el Alero del Paisano Desconocido. Además, este sitio cubre el $79 \%$ de todos aquellos desechos de talla consignados con frente de extracción preparado, sugiriendo el control en la regularización del filo. En los conjuntos de superficie, por el contrario, se evidencia el desarrollo de todas las etapas de la manufactura lítica.

\begin{tabular}{|l|l|l|l|l|l|l|l|l|}
\hline & \multicolumn{2}{|l|}{$\begin{array}{l}\text { Alero del } \\
\text { Paisano } \\
\text { Desconocido }\end{array}$} & \multicolumn{2}{l|}{$\begin{array}{l}\text { Cueva del } \\
\text { Paisano } \\
\text { Desconocido }\end{array}$} & \multicolumn{2}{l|}{ Bahía La Lancha } & \multicolumn{2}{l|}{$\begin{array}{l}\text { Laguna 4 de } \\
\text { Febrero }\end{array}$} \\
\hline Intervalos* & Largo & Ancho & Largo & Ancho & Largo & Ancho & Largo & Ancho \\
\hline $0-19.9$ & 94.6 & 95.4 & 41.1 & 52.9 & 23.5 & 17.6 & 4.49 & 5.6 \\
\hline $20-39.9$ & 4.5 & 4 & 41.1 & 47 & 47 & 58.8 & 40.4 & 67.4 \\
\hline $40-59.9$ & 0.7 & 0.5 & 11.7 & 0 & 23.7 & 17.6 & 30.3 & 21.3 \\
\hline $60-79.9$ & 0 & 0 & 5.8 & 0 & 5.8 & 5.8 & 17.9 & 3.3 \\
\hline $80-99.9$ & 0 & 0 & 0 & 0 & 0 & 0 & 4.49 & 2.2 \\
\hline $100-119.9$ & 0 & 0 & 0 & 0 & 0 & 0 & 1.12 & 0 \\
\hline $120-139.9$ & 0 & 0 & 0 & 0 & 0 & 0 & 1.12 & 0 \\
\hline
\end{tabular}

Tabla 5. Distribución porcentual de longitud y anchura de desechos de talla. *Medidos en mm.

La mayor riqueza de clases artefactuales se observa en el Alero del Paisano Desconocido y en Laguna 4 de Febrero. La bifacialidad - considerada a través de la presencia de puntas de proyectil enteras, fragmentos, preformas, bifaces y los dos FAF - es alta $(23,3 \%)$ como también lo es la representación de núcleos (20\%) (Tabla 7).

En los sitios estratificados los núcleos son pequeños y se encuentran agotados y fracturados, mientras que en superficie se han registrado piezas con potencial de reactivación y en 


\begin{tabular}{|l|l|l|l|l|}
\hline & $\begin{array}{l}\text { Alero Paisano } \\
\text { Desconocido }\end{array}$ & $\begin{array}{l}\text { Cueva Paisano } \\
\text { Desconocido }\end{array}$ & Bahía La Lancha & $\begin{array}{l}\text { Laguna 4 de } \\
\text { Febrero }\end{array}$ \\
\hline Intervalos & Espesor & Espesor & Espesor & Espesor \\
\hline $0-4.9$ & 96.4 & 47 & 23.5 & 12.3 \\
\hline $5-9.9$ & 2.7 & 47 & 41.1 & 48.3 \\
\hline $10-14.9$ & 0.5 & 5.8 & 23.5 & 26.9 \\
\hline $15-19.9$ & 0.2 & 0 & 11.7 & 7.86 \\
\hline $20-24.9$ & 0 & 0 & 0 & 3.3 \\
\hline $25-29.9$ & 0 & 0 & 0 & 1.1 \\
\hline
\end{tabular}

Tabla 6. Distribución porcentual de espesores de desechos de talla. *Medidos en mm.

\begin{tabular}{|l|l|l|l|l|l|l|l|l|}
\cline { 2 - 9 } \multicolumn{1}{c|}{} & $\begin{array}{c}\text { Laguna 4 } \\
\text { de } \\
\text { Febrero }\end{array}$ & $\begin{array}{c}\text { Médano } \\
\text { Margen } \\
\text { norte }\end{array}$ & $\begin{array}{c}\text { Península } \\
\text { Chacabuco }\end{array}$ & $\begin{array}{c}\text { Bahía } \\
\text { La } \\
\text { Lancha }\end{array}$ & $\begin{array}{c}\text { Alero del } \\
\text { Paisano } \\
\text { Desconocido }\end{array}$ & $\begin{array}{c}\text { Cueva del } \\
\text { Paisano } \\
\text { Desconocido }\end{array}$ & $\begin{array}{c}\text { Istmo } \\
\text { Pla. Cancha } \\
\text { Rayada }\end{array}$ & Total \\
\hline AFS & 0 & 0 & 0 & 1 & 0 & 2 & 0 & 3 \\
\hline Biface & 5 & 1 & 0 & 2 & 1 & 0 & 0 & 9 \\
\hline Cuchillo & 0 & 0 & 1 & 0 & 2 & 1 & 0 & 4 \\
\hline FAF & 0 & 0 & 0 & 0 & 0 & 0 & 2 & 2 \\
\hline $\begin{array}{l}\text { Punta de } \\
\text { proyectil }\end{array}$ & 1 & 2 & 1 & 4 & 3 & 0 & 0 & 11 \\
\hline Preforma & 1 & 0 & 0 & 0 & 0 & 0 & 0 & 1 \\
\hline Bola & 1 & 0 & 0 & 0 & 0 & 0 & 0 & 1 \\
\hline Núcleo & 12 & 1 & 0 & 2 & 4 & 1 & 0 & 20 \\
\hline Nódulo & 1 & 0 & 0 & 0 & 2 & 0 & 0 & 3 \\
\hline Raedera & 6 & 3 & 0 & 1 & 2 & 0 & 0 & 12 \\
\hline Raspador & 16 & 1 & 0 & 3 & 3 & 3 & 0 & 26 \\
\hline RBO & 0 & 0 & 0 & 1 & 0 & 0 & 0 & 1 \\
\hline RNCR & 3 & 1 & 0 & 1 & 2 & 1 & 0 & 8 \\
\hline $\begin{array}{l}\text { Piece } \\
\text { esquillér }\end{array}$ & 1 & 0 & 0 & 0 & 1 & 0 & 0 & 2 \\
\hline Total & 47 & 9 & 2 & 15 & 20 & 8 & 2 & 103 \\
\hline
\end{tabular}

Tabla 7 Artefactos formatizados. Ref.: AFS: artefacto de formatización sumaria; FNCR: filo natural con rastros complementarios; RBO: artefacto con retoque en bisel oblicuo.

tamaños relativamente mayores, la mitad de ellos enteros. El 40\% del total son de toba, de disponibilidad local. El sitio Laguna 4 de Febrero muestra la mayor frecuencia, diversidad de rocas y dimensiones artefactuales, muy probablemente debido al tamaño de la muestra.

En todos los sectores relevados se ha observado la presencia de un diseño artefactual particular; se trata de filos largos realizados sobre piezas bifaciales (Espinosa et al. 2007) definidas como: "Piezas bifaciales con lascados planos y penetrantes de adelgazamiento bifacial que dan lugar a un filo de sección simétrica y otro filo de sección asimétrica. Sobre el filo de sección asimétrica se regulariza el filo largo de raedera / cuchillo. El filo de sección simétrica es un filo bifacial de arista sinuosa y funciona como dorso o filo pasivo (Espinosa et al. 2013). Entre los materiales aquí analizados se han registrado 4 (3,9\%) de estos artefactos, dando cuenta de la continua distribución de este diseño en las cuencas de los lagos Tar y San Martín. Uno de ellos es una raedera que se encuentra en estratigrafía en el Alero del Paisano Desconocido, en el nivel correspondiente a 680 años AP (55-60 cm de profundidad). En otros sitios de Santa Cruz con fechados tempranos, se han hallado artefactos similares, como por ejemplo en la cueva La Martita (Aguerre 2003), ubicada en el centro-oeste de la provincia. 


\section{Discusión}

En líneas generales, las densidades artefactuales registradas en transectas son muy bajas, ajustándose a los valores obtenidos en torno a las márgenes norte y oeste del lago Tar y en la margen sur del lago San Martín (Belardi et al. 2010). Una excepción esta dada por la alta frecuencia recuperada en la transecta llevada a cabo sobre la ruta provincial $\mathrm{N}^{\circ} 31$, frente a los abrigos rocosos del Paisano Desconocido; la misma situación se observa en el porcentaje de muestreos con hallazgos (Tabla 2). Todo sugiere una baja intensidad de ocupación. Esta conclusión se ve reforzada al evaluar las tasas de depositación de artefactos por periodo de 100 años en los abrigos excavados (Tabla 8). En el Alero del Paisano Desconocido, se evidencia una diferencia cronológica que podría obedecer a la acción de un proceso de lavado de sedimento. Por este motivo se segmenta la estratigrafía en dos momentos; uno inicial, desde 4720 años AP. hasta 680 años AP., y uno posterior a partir de esta última fecha (Tabla 3). En el caso de la Cueva, debido a la ausencia de hiatos marcados, no se considera necesario subdividir la secuencia. Estas nuevas dataciones comenzaron a completar la escasa información cronológica correspondiente al Holoceno temprano y medio. Puede observarse un marcado contraste entre ambos sitios, con ocupaciones levemente solapadas en el Holoceno medio. La mayor intensidad de uso de los reparos se refleja en el momento tardío del Alero del Paisano Desconocido.

\begin{tabular}{|l|l|l|l|}
\hline \multicolumn{1}{|c|}{ Sitio } & N artefactos & $\begin{array}{c}\text { Datación } \\
\text { (años AP./ } \\
\text { periodo }\end{array}$ & $\begin{array}{c}\text { Tasa de depositación de } \\
\text { artefactos líticos (N artefactos } \\
\text { cada 100 años) }\end{array}$ \\
\hline \multicolumn{3}{|c|}{ Margen norte } \\
\hline $\begin{array}{l}\text { Cueva del Paisano } \\
\text { Desconocido }\end{array}$ & 35 & 8000 & 0,4 \\
\hline $\begin{array}{l}\text { Alero del Paisano } \\
\text { Desconocido. Inicial }\end{array}$ & 234 & $4720 / 4040$ & 5,8 \\
\hline $\begin{array}{l}\text { Alero del Paisano } \\
\text { Desconocido. Final }\end{array}$ & 421 & 680 & 61,9 \\
\hline \multicolumn{4}{|c|}{ Margen sur } \\
\hline $\begin{array}{l}\text { Campo de Bloques 1. } \\
\text { Oque dad. Inicial }\end{array}$ & 38 & $9760 / 5990$ & 0,6 \\
\hline $\begin{array}{l}\text { Campo de Bloques 1. } \\
\text { Oque dad. Fina1 }\end{array}$ & 1313 & 3770 & 34,8 \\
\hline $\begin{array}{l}\text { Campo de Bloques 2 } \\
\text { Bloque 1 }\end{array}$ & 136 & 3800 & 3,5 \\
\hline $\begin{array}{l}\text { Campo de Bloques 2 } \\
\text { Bloque 2 }\end{array}$ & 36 & 4720 & 0,7 \\
\hline
\end{tabular}

Tabla 8. Tasas de depositación artefactual comparadas entre las márgenes norte y sur del lago San Martín.

Al comparar estos valores con los obtenidos en otros sitios bajo abrigo, tales como el sitio Bloque 1 - Oquedad del Campo de Bloques 1 y los Bloques 1 y 2, del Campo de Bloques 2, ubicados en la margen sureste del lago San Martín (Belardi et al. 2010; Belardi y Campan 2009; Pallo 2009), se aprecia una tendencia similar, con tasas muy bajas en momentos iniciales y un aumento significativo hacia finales del Holoceno tardío. No obstante, en la margen sur, se evidencia una mayor profundidad temporal dado que las ocupaciones humanas se inician alrededor de 9700 años AP. (sitio Bloque 1- Oquedad) y alcanzan los inicios del 
último milenio, 950 años AP. Estas nuevas muestras de la margen norte del lago San Martín completan, en parte, un vacío en la información sobre ocupaciones tempranas posteriores a los 9700 años AP. y extienden el registro hasta momentos muy recientes (680 años AP.).

En relación con los datos paleoambientales disponibles (Bamonte 2012, Bamonte y Mancini 2009), estas dataciones son coincidentes con momentos de mayores precipitaciones, a excepción del registro más antiguo del Alero del Paisano Desconocido (4720 \pm 30 años AP.), período de menor disponibilidad hídrica. Las ocupaciones del Holoceno medio pudieron ser complementarias de aquellas registradas al este, como en el sitio Río Meseta 1. Así, se reafirma la tendencia ya observada y, además, cobra importancia para el modelo de poblamiento de áreas marginales, entre las que se incluye la zona pericordillerana.

Con respecto al análisis tecnológico, se ha observado un predominio de la obsidiana desde el inicio de las ocupaciones (margen sur del lago San Martín), a pesar de ser una roca alóctona, otorgando señales de una tecnología conservada (Belardi et al. 2010; Pallo 2009). En este sentido, cabe mencionar la recuperación en un sitio en superficie denominado Laguna 4 de Febrero de dos nódulos de obsidiana de $(80$ × 54 × 55 mm y 77 × 63 × $47 \mathrm{~mm}$ respectivamente) lo que indica su ingreso, probablemente desde la Pampa del Asador, sin modificar (Belardi et al. 2009).

La distribución de las materias primas utilizadas para la fabricación de artefactos reitera lo registrado en el resto de la cuenca; esto es, una disminución de la variación hacia el oeste. Se suma, de manera novedosa, la extensión de la circulación de toba silicificada verde (1\%), cuya cantera se localiza en la península Maipú, margen sur del lago San Martín. Las piezas de obsidiana alcanzan frecuencias altas y casi constantes y presentan una distribución similar a aquellas de limolita. Esta situación puede deberse a la disponibilidad de ambas rocas en cuencas vecinas en dirección noreste y en espacios acotados. La conjunción de estos factores permite rastrear su circulación, en este caso, hacia el suroeste.

Se ha propuesto la realización de actividades de reactivación de filos en los sitios excavados. En el caso de rocas disponibles localmente, la presencia de algunos núcleos agotados y otros con potencial de reactivación señala el desarrollo de las etapas de formatización de artefactos. Se registran artefactos con baja inversión de energía. El bajo porcentaje de fracturas se vincularía con buenas condiciones de depositación en el sur del lago San Martín (Belardi y Campan 2009; Espinosa et al. 2008; Pallo 2009).

A diferencia de los de obsidiana, los artefactos manufacturados en sílice, toba y dacita presentan una distribución más amplia. Los primeros muestran una baja formatización; en su lugar, la regularización y reactivación de filos han sido las actividades predominantes llevadas a cabo en las cuencas. Las clases artefactuales son poco diversas (núcleos, raspadores y desechos de talla, principalmente). El equipamiento de individuos parece haber sido la principal estrategia implementada (Kuhn 1995). Esta característica puede extenderse a todas las rocas silicificadas (madera silicificada, ópalo, calcedonia, jaspe). La trayectoria de los artefactos de toba y dacita indica la disponibilidad local de ambas rocas y un uso expeditivo. Por su parte, la limolita representa el 4,7\% de la muestra artefactual de la margen norte del lago San Martín, principalmente desechos de talla en el Alero del Paisano Desconocido.

Respecto de las piezas de filos largos manufacturadas sobre bifaces y/o artefactos adelgazados bifacialmente, se trata de un diseño que tiene una amplia distribución. No está limitado 
a ninguna materia prima en particular, existiendo piezas confeccionadas en dacita, ópalo, cuarzo, limolita y calcedonia. Estas rocas se presentan naturalmente en nódulos grandes.

En síntesis, la margen norte del lago San Martín expone un registro similar al de la margen sur respecto de la baja densidad artefactual en superficie y la escasa oferta de reparos rocosos, los que sugieren un uso predominantemente logístico. No se ha localizado en la margen norte un recurso particular exclusivo, como el representado por la cantera de toba silicificada verde localizada en la península Maipú, si bien su uso se ha concentrado en las inmediaciones (Espinosa et al. 2009).

\section{Conclusiones}

La información recién presentada permite incorporar a la margen norte del lago San Martín al paisaje arqueológico conocido hasta el momento. Se ha comprobado la baja intensidad de uso de este sector, consistente mayormente con actividades vinculadas con tácticas logísticas, complementarias de aquellas realizadas en el este de la cuenca.

De acuerdo con el avance de las investigaciones, se ha relevado información disponible en los espacios bajos, mostrando diferencias entre ellos (fondos de cuenca) y altos (mesetas) a nivel intra e intercuenca. Las márgenes este y sureste del lago Tar, junto con los alrededores del cerro Cach Aike, son diferentes a lo que se registra en el lago San Martín. La mayor intensidad de ocupación en los primeros puede ser interpretada en función de su ubicación próxima a vías de circulación y a una estacionalidad menos marcada. Recientes observaciones de campo, permiten postular un uso más específico de los sectores altos, destinados a la captura de presas y a la obtención de rocas y/o pigmentos. En este sentido, los sectores más occidentales que, a su vez, son aquellos que ofrecen sitios bajo abrigo (Campo de Bloques 1 y 2 -margen sur- y Alero y Cueva del Paisano Desconocido -margen norte-), habrían funcionado como campamentos operativos (Binford 1980), desde donde pudieron haberse articulado partidas logísticas hacia el bosque y las mesetas. Esta es la señal arqueológica más intensa, junto con el istmo de la península Maipú, dentro de un área marginal en el contexto del poblamiento regional (Borrero 2004).

En consecuencia, las ocupaciones correspondientes a momentos de máxima humedad a lo largo del Holoceno, principalmente en el extremo occidental de la cuenca bajo estudio, quedan incorporadas al circuito de circulación de las poblaciones cazadoras, indicando que estos espacios eran conocidos y reutilizados. Por otro lado, las ocupaciones del Holoceno tardío con mayor carácter residencial, como las relevadas sobre la margen este del lago Tar y en torno al cerro Cach Aike, estarían incluidas dentro de circuitos con mayor intensidad de circulación, tanto desde las mesetas ubicadas hacia el norte y sur como desde los espacios localizados hacia el centro-este del actual territorio de Santa Cruz.

\section{Agradecimientos}

A las familias Muruzábal, de Ea. Sierra Nevada; Díaz de las Eas. La Federica y La Lila, Andrade de Ea. Chacabuco y Hevia de Ea. Hevia por la inestimable colaboración prestada durante la realización de los trabajos de campo. Las investigaciones se llevaron a cabo en el marco de los proyectos UNPA-UARG 29A/245-1 "Ocupaciones cazadoras recolectoras en la margen norte del lago San Martín" y 29/A304 "Las cuencas de los lagos Tar y San Martín (provincia de Santa Cruz). Continuación de las investigaciones en el bosque y las mesetas circundantes e incorporación de la margen norte del lago Viedma" y del proyecto PIP-CONICET 6405 "La dinámica del poblamiento humano de las cuencas de los lagos Tar y San Martín (Pcia. de Santa Cruz) en relación 
con las variaciones ambientales del Holoceno."Una primera versión de este trabajo fue presentada en el XVIII Congreso de Arqueología Chilena (Valparaíso).

\section{Bibliografía Citada}

Aguerre, A. M.

2003 La cueva 4 de La Martita y las ocupaciones de 8000 años (Santa Cruz, Argentina). Arqueología y paleoambiente en la Patagonia santacruceña argentina, compilado por A. M. Aguerre, pp.27-61. Buenos Aires

Aschero, C.

1983 [1975] Ensayo para una clasificación morfológica de los instrumentos líticos aplicada a estudios tipológicos comparativos. Informe al CONICET.

Auer, V. y D. Cappannini 1

957 La erosión en la región de los lagos San Martín y Tar. IDIA Marzo, pp. 7-27.

Bamonte, F. y M. Mancini

2009 Características ambientales del ecotono Bosque-Estepa guante el Holoceno medio (sudoeste de Santa Cruz, Argentina). Arqueología de la Patagonia: una mirada desde el último confin, (ed. por M. Salemme, F. Santiago, M. Álvarez, E. Piana, M. Vázquez y E. Mansur), Tomo 2. pp. 881-891. Editorial Utopías, Ushuaia.

Bamonte, F.

2012 Cambios paleoecológicos y su posible relación con las ocupaciones humanas durante el Holoceno en el SO de Santa Cruz, Argentina. Tesis doctoral Universidad Nacional de Mar del Plata.

Barberena, R.

2008 Arqueología y biogeografía humana en Patagonia Meridional. Sociedad Argentina de Antropología. Colección Tesis Doctorales. Buenos Aires.

Belardi, J. B.

2005. Paisajes arqueológicos: un estudio comparativo de diferentes ambientes patagónicos. BAR International Series 1390. Oxford.

Belardi, J. B. y P. Campan

2009 Resultados arqueológicos en el Campo de Bloques 2, margen sur del lago San Martín (Provincia de Santa Cruz). Arqueología de la Patagonia: una mirada desde el último confin, (ed. por M. Salemme, F. Santiago, M. Álvarez, E. Piana, M. Vázquez y E. Mansur), Tomo 2. pp. 927-939. Editorial Utopías, Ushuaia.

Belardi, J. B., F. Carballo Marina, T. J. Bourlot y A. Re

2009 Paisajes arqueológicos, circulación e interacción en diferentes escalas: una perspectiva desde el lago Tar (Provincia de Santa Cruz). Arqueología de la Patagonia: una mirada desde el último confín, (ed. por M. Salemme, F. Santiago, M. Álvarez, E. Piana, M. Vázquez y E. Mansur), Tomo 1. pp. 219-231. Editorial Utopías, Ushuaia. 
Belardi, J. B., S. Espinosa, F. Carballo Marina, G. Barrientos, R. A. Goñi, A. Súnico, T. Bourlot, C. Pallo, A. Re y P. Campan

2010 Las cuencas de los lagos Tar y San Martín (Santa Cruz, Argentina) y la dinámica del poblamiento humano del sur de Patagonia: integración de los primeros resultados. Magallania 38(2) 137-159.

Belardi, J. B., R. A. Goñi, T. J. Bourlot y A. C. Aragone

2003. Uso del espacio y paisajes arqueológicos en la cuenca del lago Cardiel (Provincia de Santa Cruz, Argentina). Magallania 32:95-106.

Binford, L.

1980 Willow Smoke and Dog Tails: Hunter-gatherer. American Antiquity 45(1):4-20.

Bonarelli, G. y J J. Nagera

1921 Observaciones geológicas en las inmediaciones del lago San Martín (Territorio de Santa Cruz). Boletín Ministerio de Agricultura. Dirección General de Minas, Geología e Hidrología Serie B (Geología) No 27.

Borrero, L. A.

1993 Site Formation Processes in Patagonia: Depositional rates and the properties of the Archaeological Record. Explotación de Recursos Faunísticos en Sistemas Adaptativos Americanos, (comp. por J. L. Lanata) Arqueología Contemporánea 4: 107-121.

Borrero, L. A.

2004 The Archaeozoology of Andean 'Dead Ends' in Patagonia: Living near the Continental Ice Cap. Colonisation, Migration and Marginal Areas. A Zooarchaeological approach, (ed. por M. Mondini, A. S. Nuñoz y S. Wickler), pp 55-61. Oxbow Books, Oakville.

Dunnell, R. C. y W. Dancey

1983 The siteless survey: A regional scale data collection strategy. Advances in Archaeological Method and Theory, 6:267-287. Academic Press, New York.

Espinosa, S. L., J. B. Belardi y R. Molinari

2007 Análisis tecnológico de los artefactos líticos de la colección Horst Thierauf provenientes de las cuencas de los lagos Tar y San Martín (Provincia de Santa Cruz, Argentina). Arqueología de Fuego-Patagonia. Levantando piedras, desenterrando huesos... y develando arcanos, (ed. por F. Morello, M. Martinic, A. Prieto y G. Bahamonde), pp. 675- 685. Ediciones CEQUA, Punta Arenas.

Espinosa, S. L., J. B. Belardi y A. Súnico

2009 ¿Cuán al oeste? Arqueología del istmo de la península Maipú (lago San Martín, provincia de Santa Cruz) en su contexto regional. Arqueología 15: 187-205.

Espinosa, S., P. Escola y J. B. Belardi

2013. Filos largos, piezas bifaciales y diseños en la cuenca de los lagos Tar y San Martín (Santa Cruz, Argentina). Tendencias teórico-metodológicas y casos de estudio en la arqueología de Patagonia. Editado por F. Sangrando, R. Barberena, A. Gil. G. Neme, M. Giardina, L. Luna, C. Otaola, L. Paulides, L. Salgan y A. Tívoli Pp:343-350. 
Espinosa, S. L. y R. A. Goñi

1999 Viven! Una fuente de obsidiana en la Provincia de Santa Cruz. Soplando en el viento. Actas de las III Jornadas de Arqueología de la Patagonia, (ed. por el Instituto Nacional de Antropología y Pensamiento Latinoamericano y la Universidad Nacional del Comahue), pp. 177-188. Neuquén y Buenos Aires.

Kastner, S., D. Enters, Ch. Ohlendorf, T. Haberzettl, G. Kuhn, A. Lücke, Ch. Mayr, J. Reyss, S. Wastegård, B. Zolitschka

2010 Reconstructing 2000 years of hydrological variation derived from laminated proglacial sediments of Lago del Desierto at the eastern margin of the South Patagonian Ice Field, Argentina. Global and Planetary Change 72: 201-214.

Kuhn, S.

1995 Mousterian Lithic Technology. An Ecological Perspoective. Princeton University Press, Princeton.

Masiokas M.H., B.H. Luckman, R. Villalba, S. Delgado, P. Skvarca, A. Ripalta 2009 Little Ice Age fluctuations of small glaciers in the Monte Fitz Roy and Lago delDesierto areas, south Patagonian Andes, Argentina. Palaeogeography, Palaeoclimatology, Palaeoecology 281:351-362.

Pallo, C.

2009 La tecnología lítica del sitio Bloque 1-Oquedad (lago San Martín, Provincia de Santa Cruz). Resultados de la primera etapa de análisis. Arqueología de la Patagonia: una mirada desde el último confin, (ed. por M. Salemme, F. Santiago, M. Álvarez, E. Piana, M. Vázquez y E. Mansur), Tomo 1. pp. 369-381. Editorial Utopías, Ushuaia.

Rabassa, J. y A. Coronato

2002 Glaciaciones del Cenozoico Tardío. Geología y recursos naturales de Santa Cruz, (ed. por Miguel Haller), pp. 303-315. Relatorio del XV Congreso Geológico Argentino. Buenos Aires.

Stern, C.

1999 Black Obsidian from Central -South Patagonia: chemical characteristics, sources and regional distribution of artifacts. Soplando en el viento. Actas de las III Jornadas de Arqueología de la Patagonia, (ed. por el Instituto Nacional de Antropología y Pensamiento Latinoamericano y la Universidad Nacional del Comahue), pp. 221-234. Neuquén y Buenos Aires. 